\title{
Optimal design criteria for isolation devices in vibration control
}

\author{
Giuseppe Carlo Marano $\$$ and Sara Sgobba* \\ §Technical University of Bari, DIASS, viale del Turismo 10, \\ 74100 Taranto (Italy) \\ *International Telematic University UNINETTUNO \\ Corso Vittorio Emanuele II, 39 \\ 00186 Roma (ITALY)
}

\begin{abstract}
Vibration control and mitigation is an open issue in many engineering applications. Passive strategies was widely studied and applied in many contests, such as automotive, aerospatial, seismic and similar. One open question is how to choose opportunely devices parameters to optimize performances in vibration control. In case of isolators, whose the main scope is decoupling structural elements from the vibrating support, optimal parameters must satisfy both vibration reduction and displacement limitation.

This paper is focused on the a multi-objective optimization criterion for linear viscous-elastic isolation devices, utilised for decreasing high vibration levels induced in mechanical and structural systems, by random loads. In engineering applications base isolator devices are adopted for reducing the acceleration level in the protected system and, consequently, the related damage and the failure probability in acceleration sensitive contents and equipment. However, since these devices act by absorbing a fraction of input energy, they can be subjected to excessive displacements, which can be unacceptable for real applications. Consequently, the mechanical characteristics of these devices must be selected by means of an optimum design criterion in order to attain a better performance control.

The proposed criterion for the optimum design of the mechanical characteristics of the vibration control device is the minimization of a bi-dimensional objective function, which collects two antithetic measures: the first is the index of device efficiency in reducing the vibration level, whereas the second is related to system failure, here associated, as in common applications, to the first exceeding of a suitable response over a given admissible level.

The multi-objective optimization will be carried out by means of a stochastic approach: in detail, the excitation acting at the support of the protected system will be assumed to be a stationary stochastic coloured process.

The design variables of optimization problem, collected in the design vector $(D V)$, are the device frequency and the damping ratio. As cases of study, two different problems will be analysed: the base isolation of a rigid mass and the tuned mass damper positioned on a MDoF structural system, subject to a base acceleration.
\end{abstract}


The non dominated sorting genetic algorithm in its second version (NSGA-II) is be adopted in order to obtain the Pareto sets and the corresponding optimum $D V$ values for different characterizations of system and input.

Keywords: Random vibrations, multi-objective stochastic optimization, base isolator, tuned mass damper, genetic algorithm.

\section{Introduction}

Dynamic actions are nowadays a wide engineering topic in many applicative and research areas, such as automotive, civil and aerospace. One main problem is how properly model dynamic actions, because of there are many real conditions where it is practically impossible to accurate predict future dynamic actions (i.e. earthquakes, wind pressure, sea waves and rotating machinery induced vibrations). In those cases external loads can be suitably modelled only by using random processes, and as direct consequence, also systems responses are random processes. In these environments, random dynamic analysis seems to be the most suitable method to get practical information concerning systems response and reliability (see for example [1]). It is obvious that also structural optimization methods seem to be practically approached by means of random vibrations theory. Concerning this problem, some recent works have been proposed, typically based on Standard Optimization Problem (SOP), which finds the optimum solution that coincides with the minimum or the maximum value of a scalar Objective Function (OF). The first problem definition of structural optimization was proposed by [2], in which constraints were defined by using probabilistic indices of the structural response and the $O F$ was defined by the structural weight, leading to a standard nonlinear constrained problem.

In the field of seismic engineering, the use of a stochastic defined $O F$ has been proposed for the optimum design of the damping value of a vibrations control device placed on the first story of a building [3], and was defined by the maximum displacement under a white noise excitation. A specific and more complete stochastic approach has also been proposed by [4], aimed to stiffness-damping simultaneous optimization of structural systems. In this work the sum of system response mean squares due to a stationary random excitation was minimized under constraints on total stiffness capacity and total damping capacity.

More recently, an interesting stochastic approach for optimum design of damping devices in seismic protection has been proposed by [5], aimed to minimize the total building life-cycle cost. It was based on a stochastic dynamic approach for failure probability evaluation, and the $O F$ was defined in a deterministic way. The optimization problem was formulated by adopting as design variables the location and the amount of the viscous elastic dampers, adopting as constraints the failure probability associated to the crossing of the maximum inter-storey drift over a given allowable value. Reliability analysis was developed by means of the application of the first crossing theory in stationary conditions.

Another interesting work in the field of stochastic structural optimization regards the unconstrained optimization of single [6] and multiple [7] tuned mass dampers, by using as $O F$ the structural displacement covariance of the protected system and modelling the input by means of a stationary white noise process.

However, the SOP does not usually hold correctly many real structural problems, where often different and conflicting objectives may exist. In these situations, the SOP is utilized by selecting a single objective and then incorporating the other objectives as constraints. The 
main disadvantage of this approach is that it limits the choices available to the designer, making the optimization process a rather difficult task.

Instead of unique SOP solution, a set of alternative solutions can be usually achieved. They are known as the set of Pareto optimum solutions, and represent the best solutions in a wide sense, that means they are superior to other solutions in the search space, when all objectives are considered. If any other information about the choice or preference is given, no one of the corresponding trade-offs can be said to be better than the others. Many works in last decade have been done by different authors in the field of multi-objective structural optimization, for systems subject to static or dynamic loads [8].

This work deals with a multi-objective optimization of linear viscous-elastic devices, which are introduced in structural and mechanical systems in order to reduce vibrations level induced by random actions applied at the support. As application, two different problems are considered: first, the vibration base isolation of a rigid mass subject to support acceleration. In detail this is the problem of a vibration absorber for a rigid element isolated from a vibrating support, subject to a random acceleration process. This represents a typical application in many real problems, in mechanical, civil and aeronautics engineering. The main system is a rigid mass linked with the support by means of a linear viscous-elastic element (fig.1). In the multi-objective optimization, the $O F$ is a vector which contains two elements: the first one is an index of device performance in reducing the vibration level, here expressed by the acceleration reduction factor. This is assumed to be, in stochastic meaning, the ratio between the mass and the support acceleration variances.

The second objective function is the displacement of the protected mass. In probabilistic meaning it is obtained in terms of the maximum displacement which will not be exceeded in a given time interval and with a given probability. This is achieved by adopting the threshold crossing probability theory. Design variables, which are assumed to be the isolator damping ratio $\xi_{S}$ and its pulsation $\omega_{s}$, are collected in the design vector $(D V)$. The support acceleration is modelled as a filtered stationary stochastic process.

In order to obtain the Pareto set in the two dimensions space of OFs, and the optimum solution in the space of design variables, a specific genetic algorithm approach (the NSGA-II one) is adopted in the two cases of study. A sensitive analysis on the optimum solution is finally performed under different environmental conditions.

\section{Multi-objective stochastic optimization of random vibrating systems}

The proposed stochastic multi-objective optimization criterion is adopted in this study in order to define the optimum mechanical parameters in classical problems of vibration control. As before mentioned, two applications are considered which regard, in general, the limitation of vibration effects in mechanical and structural systems subject to base accelerations.

The optimization problem could be formulated as the search of design parameters, collected in the Design Vector $(D V) \mathbf{b}$, defined in the admissible domain $\boldsymbol{\Omega}_{\mathbf{b}}$, able to minimize a given OF. This problem, in general, can be formulated in a standard deterministic way, or in a stochastic one, for example by means of response spectral moments. This approach, as before mentioned, has anyway some limits, because when designer looks for the optimum solution, he has to face with the selection of the most suitable criterion for measuring 
performance. It is evident that many different quantities, which have a direct influence on the performance, can be considered as efficient criteria. At the same time, those quantities which must satisfy some imposed requirements, and cannot be assumed as criteria, are then used as constraints. It is common in optimization problems, therefore, to use a single $O F$ subjected to some probabilistic constraints, as in the first stochastic optimization problem [2]. Usually, inequality constraints on system failure probability are utilised.

In the multi-objective formulation the conflict which may or may not exist between the different criteria is an essential point. Only those quantities which are competing should be considered as independent criteria. The others can be combined into a single criterion, which represents the whole group.

\section{Case of study: protection of a rigid mass from a vibrating support}

Let us consider first the case of the isolation of a rigid mass positioned on a vibrating support. In engineering applications the mass can represent a subsystem located on a vibrating mechanical support, as motor device, airplane structure, seismic isolated building and similar. In all these situations, the main goal is to limit the induced accelerations and to control the displacement of the rigid mass with respect to the support. The first objective is related to excessive inertial forces transmitted for example to electronic or mechanical devices, which can be sensitive to this effect (i.e. acceleration sensitive contents and equipment). The second objective is related to an excessive displacement of the protected mass, which can become unacceptable, for example, if the system is located quite closer to other elements, or if the vibration isolator has a limited acceptable lateral deformation over which it will collapse.

The protected element is modelled as a rigid body having a mass $m$. The isolator device is modelled as a simple viscous-elastic element, which connects the vibrating base with the supported mass (Fig. 1).

\section{Protected element}

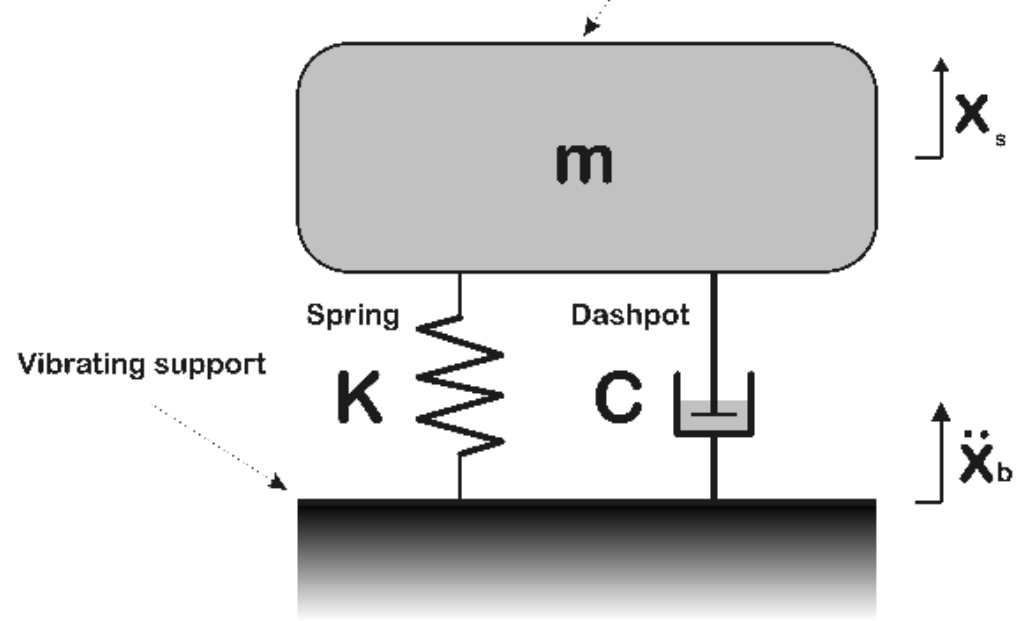

Fig. 1. Schematic Model of a rigid mass isolated from a vibrating support by means of an isolation device. 
The stiffness $k$ and the damping $c$ of the isolator device must be optimized in order to minimize the vibration effects on the rigid mass $m$.

The base acceleration is a stochastic coloured process $\ddot{X}_{b}(t)$ modelled by means of a second order linear filter [9]:

$$
\ddot{X}_{b}(t)=\ddot{X}_{f}(t)+w(t)=-\left(2 \xi_{f} \omega_{f} \dot{X}_{f}+\omega_{f}^{2} X_{f}\right)
$$

where $w(t)$ is a stationary Gaussian zero mean white noise process, $\omega_{f}$ is the filter pulsation and $\xi_{f}$ is the filter damping ratio. The motion equations of this combined system are:

$$
\begin{gathered}
\ddot{X}_{s}(t)+2 \xi_{s} \omega_{s} \dot{X}_{s}+\omega_{s}^{2} X_{s}=-\ddot{X}_{b} \\
\ddot{X}_{f}(t)+2 \xi_{f} \omega_{f} \dot{X}_{f}+\omega_{f}^{2} X_{f}=-w(t) \\
\ddot{X}_{b}(t)=\ddot{X}_{f}+w(t)
\end{gathered}
$$

In the space equations (2)-(4) can be written as:

$$
\dot{\mathbf{Z}}=\mathbf{A} \mathbf{Z}+\mathbf{F}
$$

where the space vector is:

$$
\mathbf{Z}=\left(\begin{array}{llll}
X_{s} & X_{f} & \dot{X}_{s} & \dot{X}_{f}
\end{array}\right)^{T}
$$

and the system matrix is:

$$
\mathbf{A}=\left(\begin{array}{cccc}
0 & 0 & 1 & 0 \\
0 & 0 & 0 & 1 \\
-\omega_{s}^{2} & \omega_{f}^{2} & -2 \xi_{s} \omega_{s} & 2 \xi_{f} \omega_{f} \\
0 & -\omega_{f}^{2} & 0 & -2 \xi_{f} \omega_{f}
\end{array}\right)
$$

where:

$$
\omega_{s}=\sqrt{\frac{k}{m}} ; \xi_{s}=\frac{c}{2 \sqrt{k m}}
$$

Finally, the input vector is: 


$$
\mathbf{F}=-\left(\begin{array}{llll}
0 & 0 & 0 & w(t)
\end{array}\right)^{T}
$$

The space state covariance matrix $\mathbf{R}_{\mathbf{Z Z}}=\left\langle\mathbf{Z} \mathbf{Z}^{T}\right\rangle$ is obtained by solving the Lyapunov equation:

$$
\mathbf{A R}_{\mathbf{z Z}}+\mathbf{R}_{\mathbf{z Z}} \mathbf{A}^{T}+\mathbf{B}=\mathbf{0}
$$

The variance $\sigma_{\ddot{y}_{s}}^{2}$ of the absolute mass acceleration $\ddot{y}_{s}=\ddot{x}_{s}+\ddot{x}_{b}$ is:

where:

$$
\sigma_{\ddot{y}}^{2}=\mathbf{D}^{T} \mathbf{R}_{\mathbf{z z}} \mathbf{D}
$$

$$
\mathbf{D}=\left(\begin{array}{llll}
-\omega_{s}^{2} & 0 & -2 \xi_{s} \omega_{s} & 0
\end{array}\right)^{T}
$$

\section{Formulation of multi-objective optimization of device mechanical characteristics}

The multi-objective stochastic optimization problem concerns the evaluation of $D V$ $\mathbf{b}=\left(\omega_{s}, \xi_{s}\right)$ which is able to satisfy the reduction of the transmitted inertial acceleration in the rigid mass and to limit the displacement of this one with respect to the support. These two criteria conflict each others because, when the support rigidity grows at that time the acceleration reduction (i.e. the performance) and the lateral displacement decrease. This situation corresponds for example to the design of a well known vibration control device utilized in the field of seismic engineering: the base isolator. The decoupling between the vibrating support and the protected element, i.e. the effectiveness of vibration control strategy, increases monotonically with the reduction of device stiffness, but at the same time the device displacement grows up. Therefore, in the design of these devices the level of reduction of transmitted acceleration in the protected element, (i.e. the efficiency of control strategy) is related to the allowable maximum value of device displacement, and therefore these two conflicting criteria must be considered in the design.

The multi-objective optimization problem is finally posed:

$$
\min \left\{O F_{1}, O F_{2}\right\}
$$

In detail:

$$
O F_{1}(\mathbf{b})=\left(\frac{\sigma_{\ddot{y}_{S}}(\mathbf{b})}{\sigma_{\ddot{x}_{b}}}\right)
$$

where the base vibrating acceleration variance is [11]: 


$$
\sigma_{\ddot{x}_{b}}^{2}=\frac{\pi}{2} \frac{S_{0} \omega_{f}}{\xi_{f}}\left(1+4 \xi_{f}^{2}\right)
$$

being $S_{0}$ the power spectral density function of the white noise process.

This $O F$ is a direct protection efficiency index: it tends to a null value for a totally systembase decoupling, and tends to unit for a system rigidly connected with the vibrating base, and so subject to the same acceleration $\sigma_{\ddot{x}_{b}}$.

In order to make explicit the $O F_{2}$, the maximum displacement value $X_{s}^{\text {max }}$ that will not be exceeded with a given probability $\widetilde{P}_{f}$ in an assigned time interval (assumed to be the duration of random vibration $T$ ) is adopted. Therefore:

$$
O F_{2}(\mathbf{b})=X_{S}^{\max }(\mathbf{b}): P_{f}\left(X_{S}^{\max }, \mathbf{b}\right)=P\left\{\left|X_{S}\right| \geq X_{S}^{\max }(\mathbf{b}) \mid t \in[0, T]\right\} \leq \widetilde{P}_{f}
$$

In case of rare failure events, the Poisson hypothesis could be reasonably utilised and so [1]:

$$
P_{f}\left(X_{S}^{\max }, \mathbf{b}\right)=1-e^{-v\left(X_{S}^{\max }, \mathbf{b}\right) T}
$$

where the unconditioned mean crossing rate is:

$$
v=\left(X_{S}^{\max }, \mathbf{b}\right)=\frac{1}{\pi} \frac{\sigma_{\dot{X}_{S}}}{\sigma_{X_{S}}} e^{-\left\{\frac{1}{2}\left(\frac{X_{S}^{\max }}{\sigma_{X_{S}}}\right)^{2}\right\}}
$$

Finally one obtains:

$$
O F_{2}(\mathbf{b})=X_{S}^{\max }=\sqrt{-2 \sigma_{X_{S}}^{2} \ln \left(-\frac{\pi}{T} \frac{\sigma_{X_{S}}}{\sigma_{\dot{X}_{S}}} \ln \left(1-\widetilde{P}_{f}\right)\right)}
$$

The two objective functions are plotted in Figure 2 in terms of ratio $\omega_{s} / \omega_{f}$ and $\xi_{s}$. 

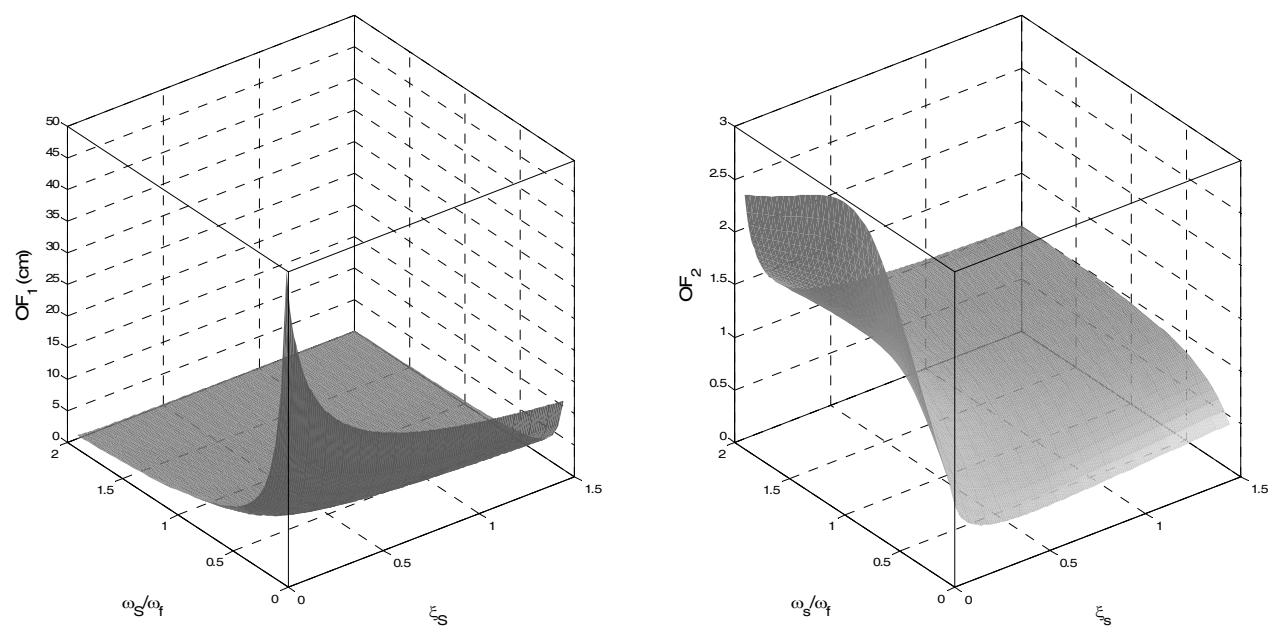

Fig. 2. Conflicting aspect of the two proposed objective functions.

\section{An overview on methods for multi-objective optimization using gas}

Many real engineering problems often involve several OFs each other in conflict and for them it is not possible to define an universally approved criteria of "optimum" as in single objective optimization. In this field, instead of aiming to find a single solution one can try to produce a set of good compromises. In a typical minimization-based $M O O P$, given two candidate solutions $\left\{\mathbf{b}_{j}, \mathbf{b}_{k}\right\}$, if:

$$
\forall i \in\{1, \ldots, M\}, O F_{i}\left(\mathbf{b}_{j}\right) \leq O F_{i}\left(\mathbf{b}_{k}\right) \wedge \exists i \in\{1, \ldots, M\}: O F_{i}\left(\mathbf{b}_{j}\right)<O F_{i}\left(\mathbf{b}_{k}\right)
$$

and defined the two objective vectors:

$$
\begin{aligned}
& \mathbf{v}\left(\mathbf{b}_{j}\right)=\left\{O F_{1}\left(\mathbf{b}_{j}\right), \ldots, O F_{M}\left(\mathbf{b}_{j}\right)\right\} \\
& \mathbf{v}\left(\mathbf{b}_{k}\right)=\left\{O F_{1}\left(\mathbf{b}_{k}\right), \ldots, O F_{M}\left(\mathbf{b}_{k}\right)\right\}
\end{aligned}
$$

the vector $\mathbf{v}\left(\mathbf{b}_{j}\right)$ is said to dominate vector $\mathbf{v}\left(\mathbf{b}_{k}\right)$ (denoted by $\mathbf{v}\left(\mathbf{b}_{j}\right) \prec \mathbf{v}\left(\mathbf{b}_{k}\right)$ ). Moreover, if no feasible solution, $\mathbf{v}\left(\mathbf{b}_{k}\right)$, exists that dominates solution $\mathbf{v}\left(\mathbf{b}_{j}\right)$, then $\mathbf{v}\left(\mathbf{b}_{j}\right)$ is classified as a non-dominated or Pareto optimal solution. The collection of all Pareto optimal solutions are known as the Pareto optimal set or Pareto efficient set, instead the 
corresponding objective vectors are described as the Pareto front or Trade-off surface. Unfortunately, the Pareto optimum concept almost does not give a single solution, but a set of possible solutions, that cannot be used directly to find the final design solution by an analytic way. On the contrary, usually the decision about the "best solution" to be adopted is formulated by so-called (human) decision maker (DM), while rarely DM doesn't have any role and a generic Pareto optimal solution is considered acceptable (no - preference based methods). On the other hand, several preference-based methods exist in literature. A more general classification of the preference-based method is considered when the preference information is used to influence the search [12]. Thus, in a priori methods, DM's preferences are incorporated before the search begins: therefore, based on the $D M^{\prime} s$ preferences, it is possible to avoid producing the whole Pareto optimal set. In progressive methods, the DM's preferences are incorporated during the search: this scheme offers the sure advantage to drive the search process but the DM may be unsure of his/her preferences at the beginning of the procedure and may be informed and influenced by information that becomes available during the search. A last class of methods is a posteriori: in this case, the optimiser carries out the Pareto optimal set and the DM chooses a solution ("searches first and decides later"). Many researchers view this last category as standard so that, in the greater part of the circumstances, a MOOP is considered resolved once that all Pareto optimal solutions are recognized. In the category of a posteriori approaches, different Evolutionary Algorithms $(E A)$ are presented. In [13] an algorithm for finding constrained Pareto-optimal solutions based on the characteristics of a biological immune system (Constrained Multi-Objective Immune Algorithm, CMOIA) is proposed. Other diffused algorithms are the Multiple Objective Genetic Algorithm (MOGA) [14] and the Non dominated Sorting in Genetic Algorithm (NSGA) [15]. In this work the NSGA-II [16] will be adopted in order to obtain the Pareto sets and the correspondent optimum $D V$ values for different systems and input configurations, for both the analysed problems (the vibration base isolation of a rigid mass and the TMD positioned on MDoF system subject to a base acceleration). Particularly, the Real Coded GA [17], Binary Tournament Selection [18], Simulated Binary Crossover (SBX) [19] and polynomial mutation [17] are used.

\section{Multi-objective optimization of isolator mechanical characteristics}

In this section the results of this first optimization problem are analysed. It is assumed that the admissible domain for $\mathbf{b}$ is the following:

$$
\mathbf{\Omega}_{\mathbf{b}}=\left\{\xi_{s}, \omega_{s}: 0.01 \leq \xi_{s} \leq 2.5 \vee 1 \mathrm{rad} / \mathrm{sec} \leq \omega_{s} \leq 30 \mathrm{rad} / \mathrm{sec}\right\}
$$

System parameters are listed in table 1. 


\begin{tabular}{llll}
\hline Filter damping ratio $\xi_{f}$ & 0.6 & \\
Filter pulsation $\omega_{f}$ & 20.94 & $(\mathrm{rad} / \mathrm{sec})$ \\
Power spectral density $S_{0}$ & 1000 & $\mathrm{~cm}^{2} / \mathrm{sec}^{3}$ \\
$T$ & $\sim$ & $10^{3}$ & $\mathrm{sec}$ \\
Max probability of failure $P_{f}$ & $10^{-2}$ & \\
\hline
\end{tabular}

Table 1. System parameters.

Concerning NDGA-II setup, after several try and error analyses, the parameters reported in table 2 have been adopted for the analysis. The selection derives from considerations about the equilibrium of computing cost and solution stability. The population size has been chosen as 500 in order to obtain a continuum Pareto front, and the maximum iteration number here used (100) has been determined after several numerical experiments (type try and error) which indicated that it is the minimum value to obtain stable solutions. This means that adopting a smaller iterations number, some differences in Pareto fronts (obtained for the same input data) take place.

\begin{tabular}{lc}
\hline Maximum generation & 500 \\
\hline Population size & 100 \\
\hline Crossover probability & 0.9 \\
\hline Mutation probability & 0.1 \\
\hline
\end{tabular}

Table 2. NDGA-II setup.

\begin{tabular}{|c|c|c|c|c|}
\hline Symbols & $\mathrm{OF}_{2}(\mathbf{c m})$ & $\mathrm{OF}_{1}(\mathbf{c m})$ & $\omega_{S}^{\text {opt }}(\mathbf{r a d} / \mathbf{s e c})$ & $\xi_{S}^{\text {opt }}$ \\
\hline $\mathbf{O}$ & 171.3159 & 0.2227 & 1 & 0.6256 \\
\hline $\mathbf{\square}$ & 39.5099 & 0.3896 & 2.7629 & 0.7276 \\
\hline $\mathbf{0}$ & 110.5646 & 0.2624 & 1.3313 & 0.6910 \\
\hline & 1.7741 & 0.9402 & 17.8002 & 2.1599 \\
\hline
\end{tabular}

Table 3. Some numerical data from figure 3. 


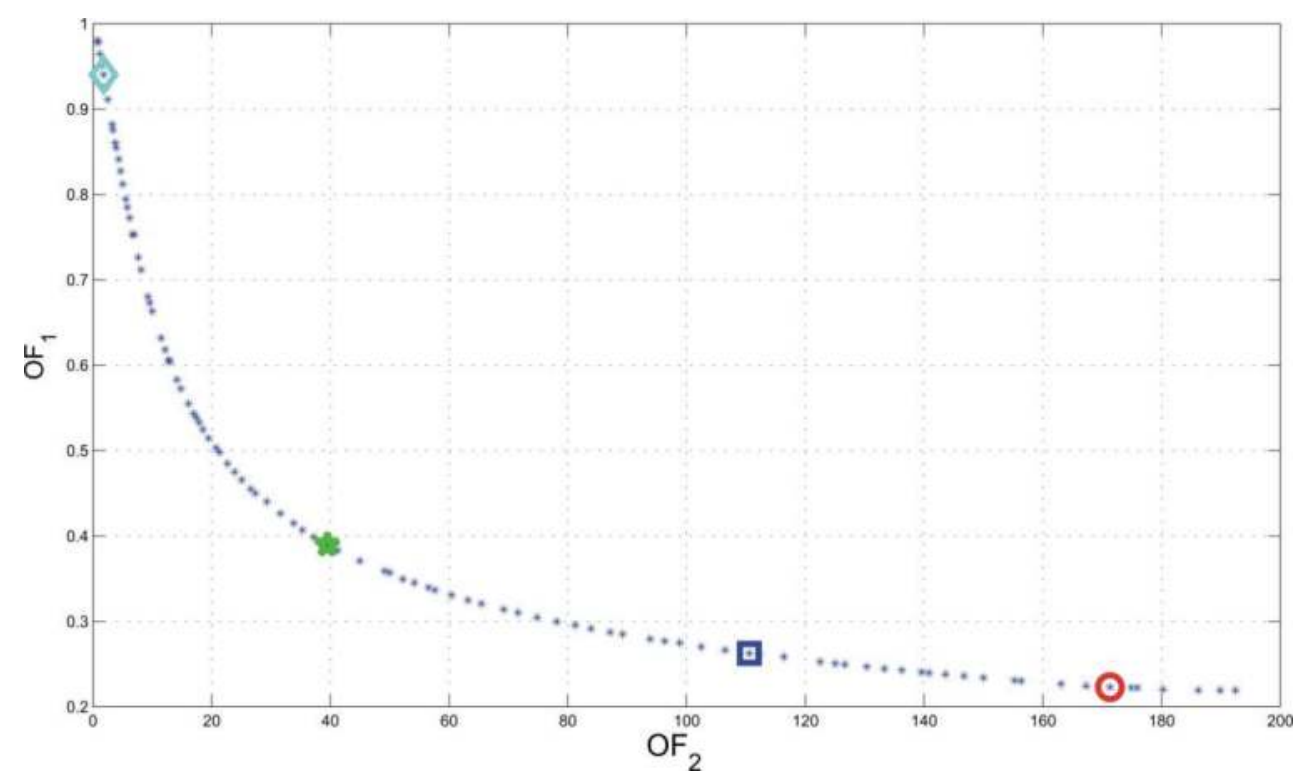

Fig. 3. Pareto front.

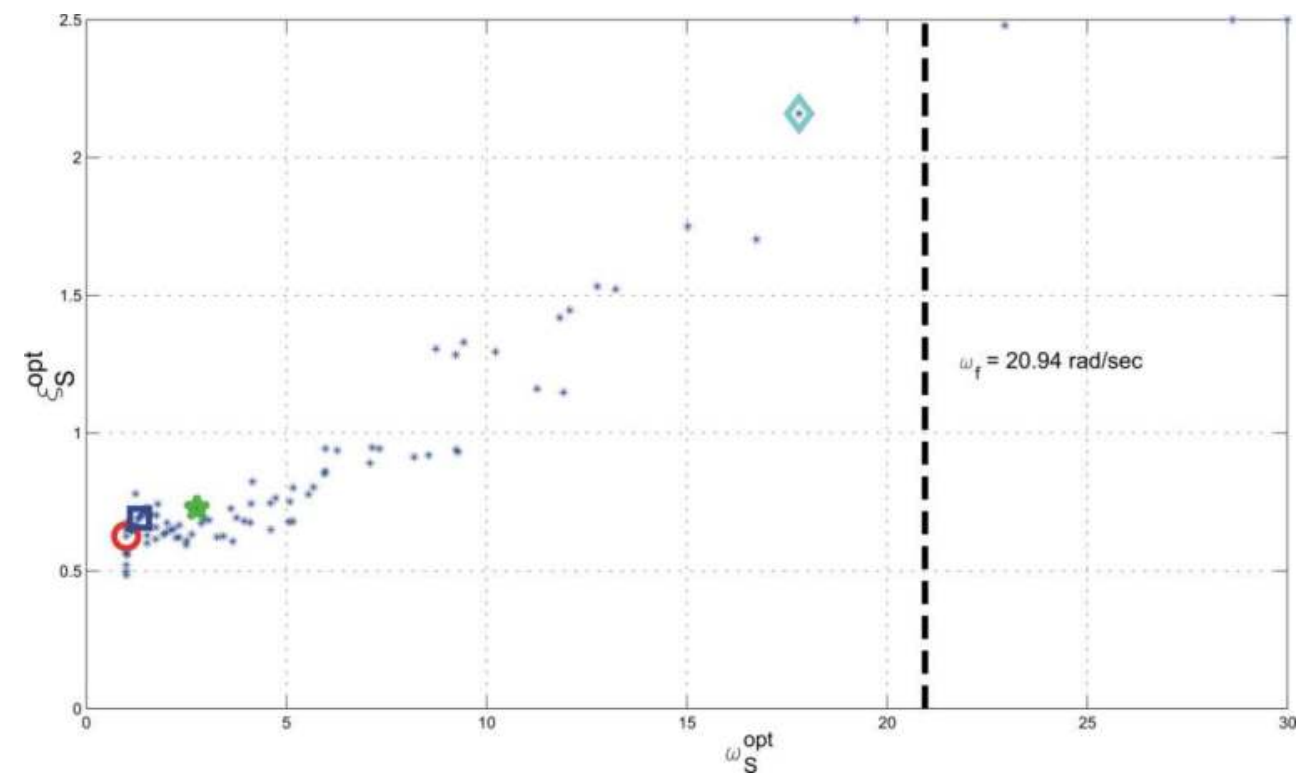

Fig. 4. Space of DV elements.

Figures 3 and 4 show the Pareto front and the space of $D V$ elements, respectively, in this first case of multi-objective optimization problem. More precisely, in figure 4 on $\mathrm{X}$-axis the 
optimum frequency of the device $\omega_{s}^{\text {opt }}$ is plotted, whereas on the Y-axis the optimum damping ratio $\xi_{T}^{o p t}$ is shown. The vertical line corresponds to the filter frequency $\omega_{f}$. In Table 3 some numerical data derived from these figures are also reported.

From figure 3 first of all it is possible to notice that a larger level of protection is related to an increase of allowable displacement. Anyway an asymptotic limit value of performance exists, that means that the reduction of transmitted acceleration is in the analysed example at least about 0.2. Moreover, some interesting observations can be carried out by observing the slope of Pareto front, which is not a convex curve. It is possible to distinguish three different portions of the Pareto front, which correspond to different criteria in using the vibration control strategy. In fact, on the left section of the Pareto front, which is related to a low efficiency, by means of a little grow of maximum allowable displacement one can obtain a large increase of performance (the slope is high). Then, in the second portion of Pareto set, the slope of the front reduces and, finally, in the right part an increase of performance is obtained only by means of a large increase of maximum admissible displacement. In this last situation, only little variations of optimum design variables take place (fig. 4). On the contrary, the reduction of maximum displacement is reached by increasing both frequency and damping. The variation is fast as the displacement reduces. Moreover, if the imposed displacement is very low, the control strategy acts by increasing the system frequency and by increasing quickly also the damping, which is associated to energy dissipation.

Figures 5, 7 and 9 show different Pareto fronts obtained for different values of power spectral density, filter damping ratio and filter pulsation. Figures 6, 8 and 10 show the corresponding optimum design variables. All the other parameters adopted are the same of figure 3 .

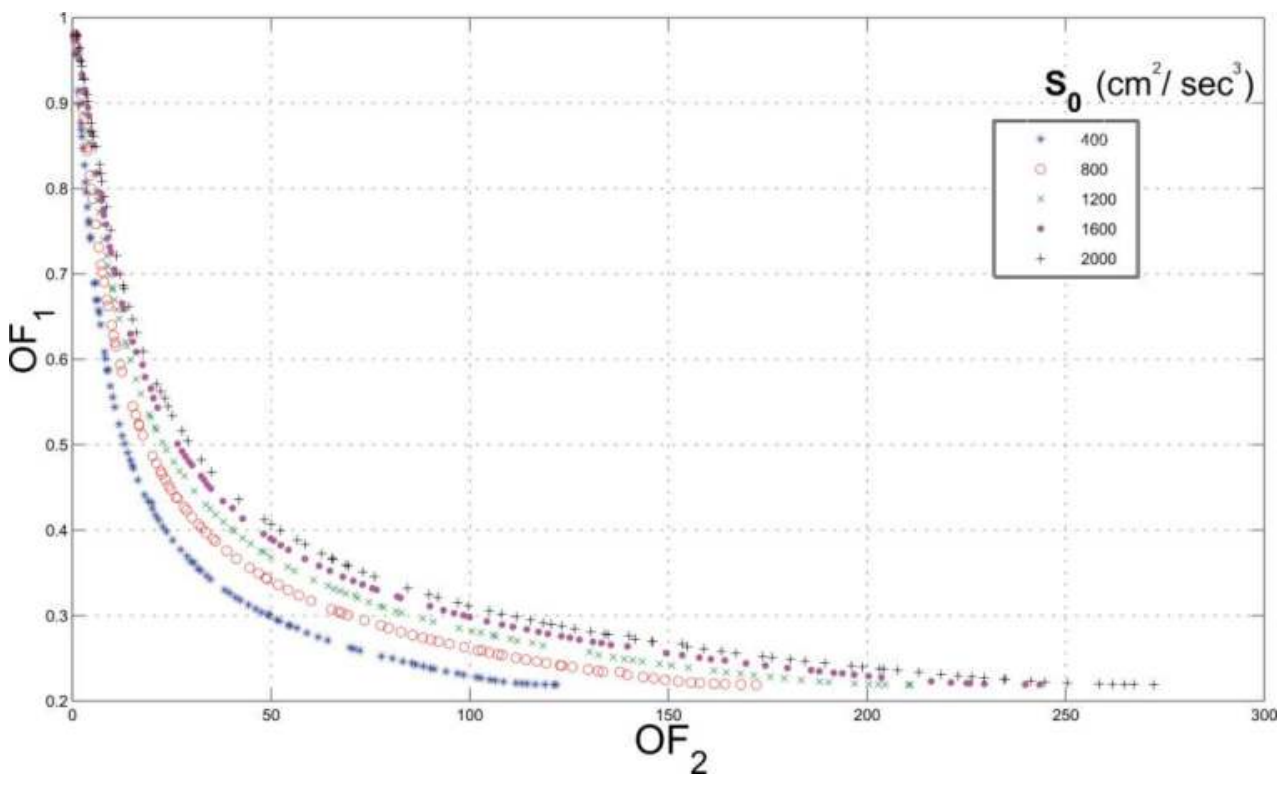

Fig. 5. Sensitivity of Pareto front for different values of power spectral density. 


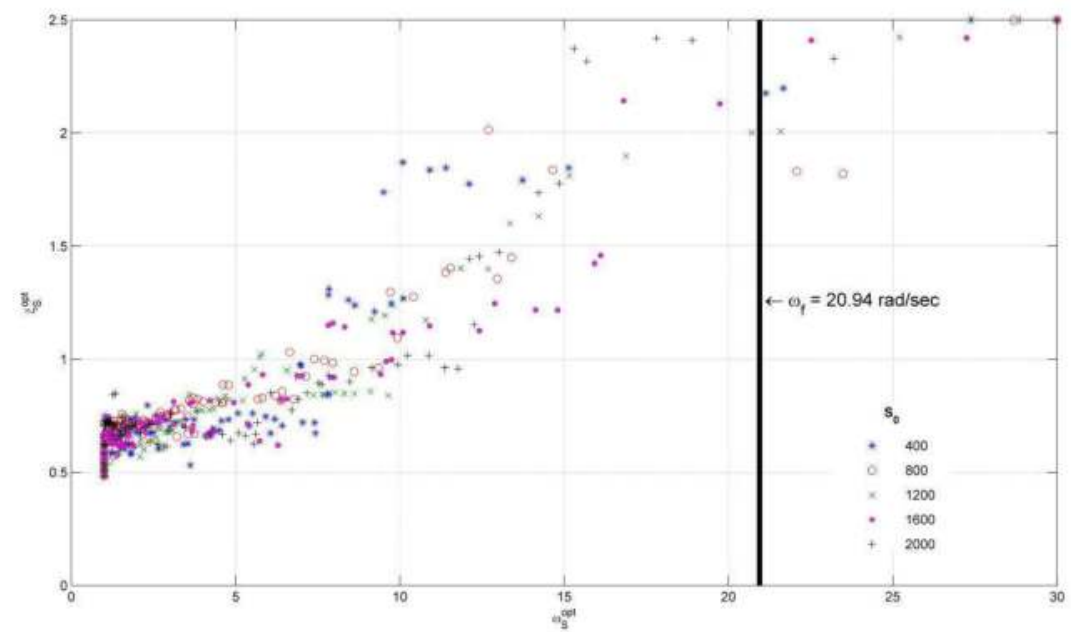

Fig. 6. Space of DV elements of multi-objective problem for different values of power spectral density.

With reference to figure 5 it is possible to notice that a variation of power spectral density induces variation of optimum Pareto front, due to non-linearity of $O F_{2}$. It is evident that higher performances are associated with low values of $S_{0}$, but the maximum level of vibration reduction (expressed by the asymptotic value of $O F_{1}$ ) is about the same in all cases, also if this situation corresponds to larger displacements for higher values of $S_{0}$. This outcome is quite clear, because the requirement on the maximum displacement is associated to $S_{0}$ by means of a non-linear formulation; meanwhile the vibration reduction is a linear function of this parameter.

However, the strategy adopted for the optimal solution in terms of design variables are about the same for all values of $S_{0}$, as shown in figure 6 , where the same variability of the Pareto set for all values of $S_{0}$ can be observed. 


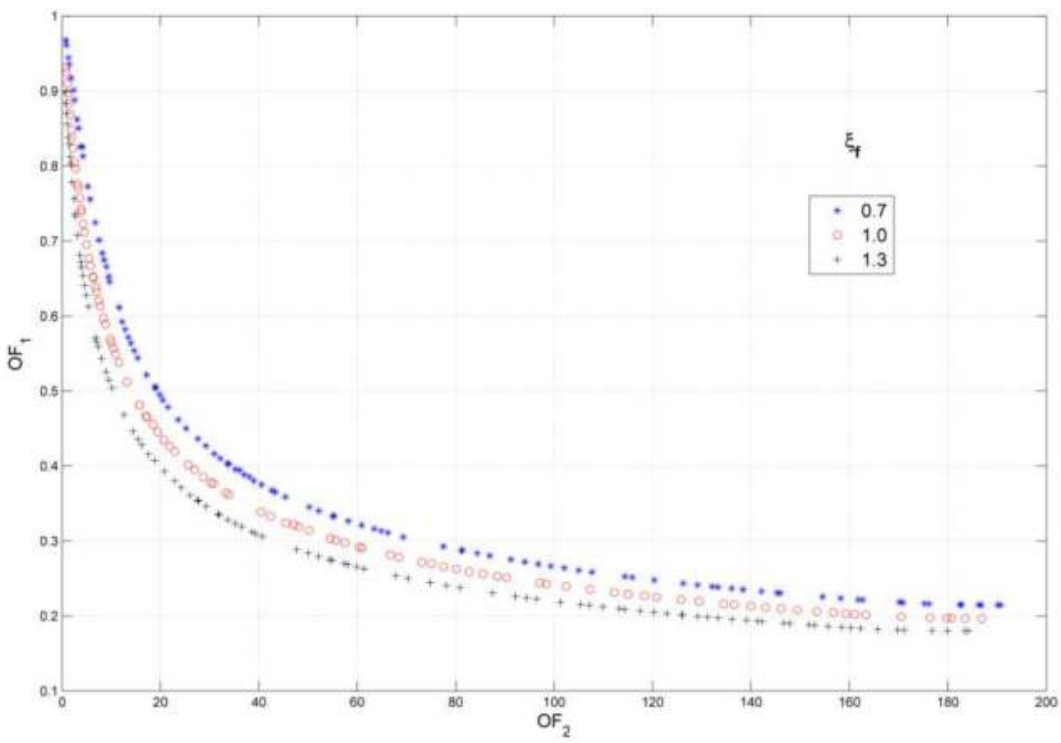

Fig. 7. Sensitivity of Pareto front for different values of filter damping ratio.

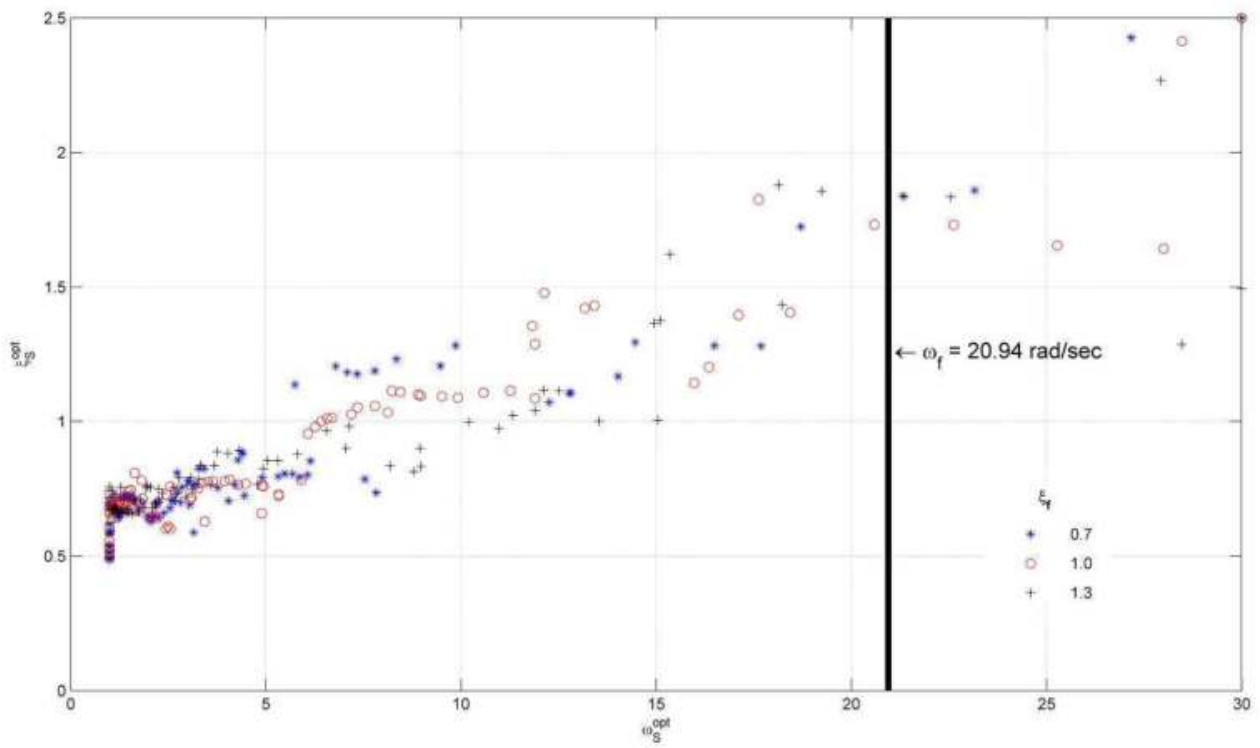

Fig. 8. Space of DV elements of multi-objective problem for different values of filter damping ratio. 


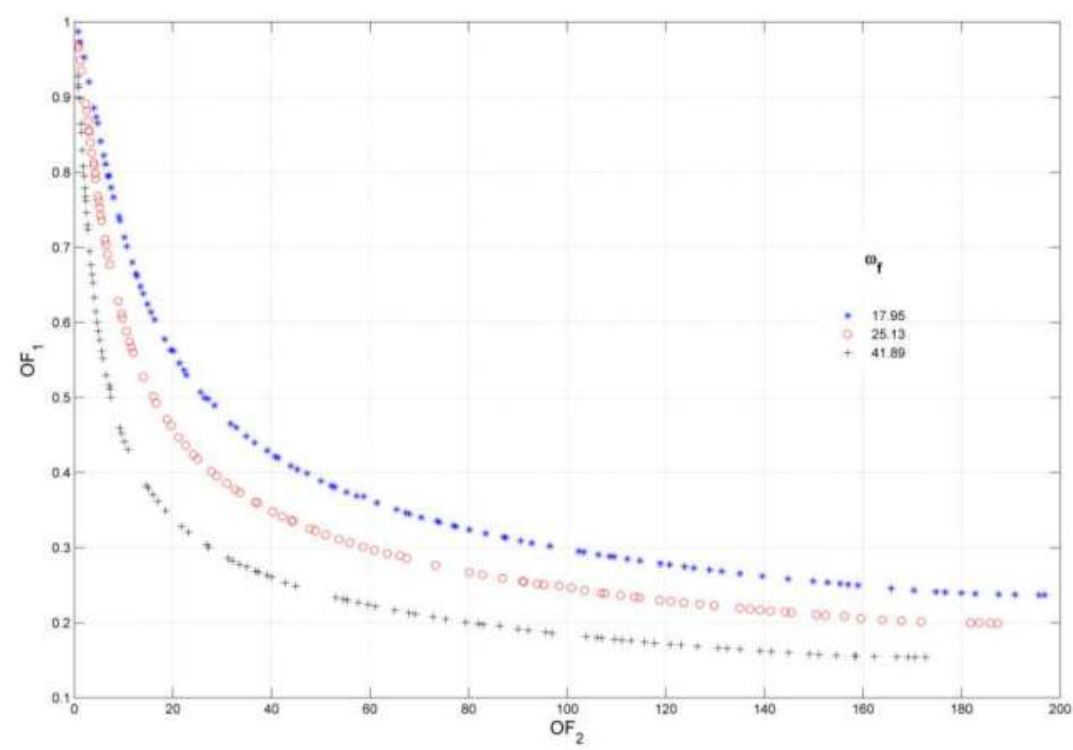

Fig. 9. Pareto front for different values of filter pulsation.

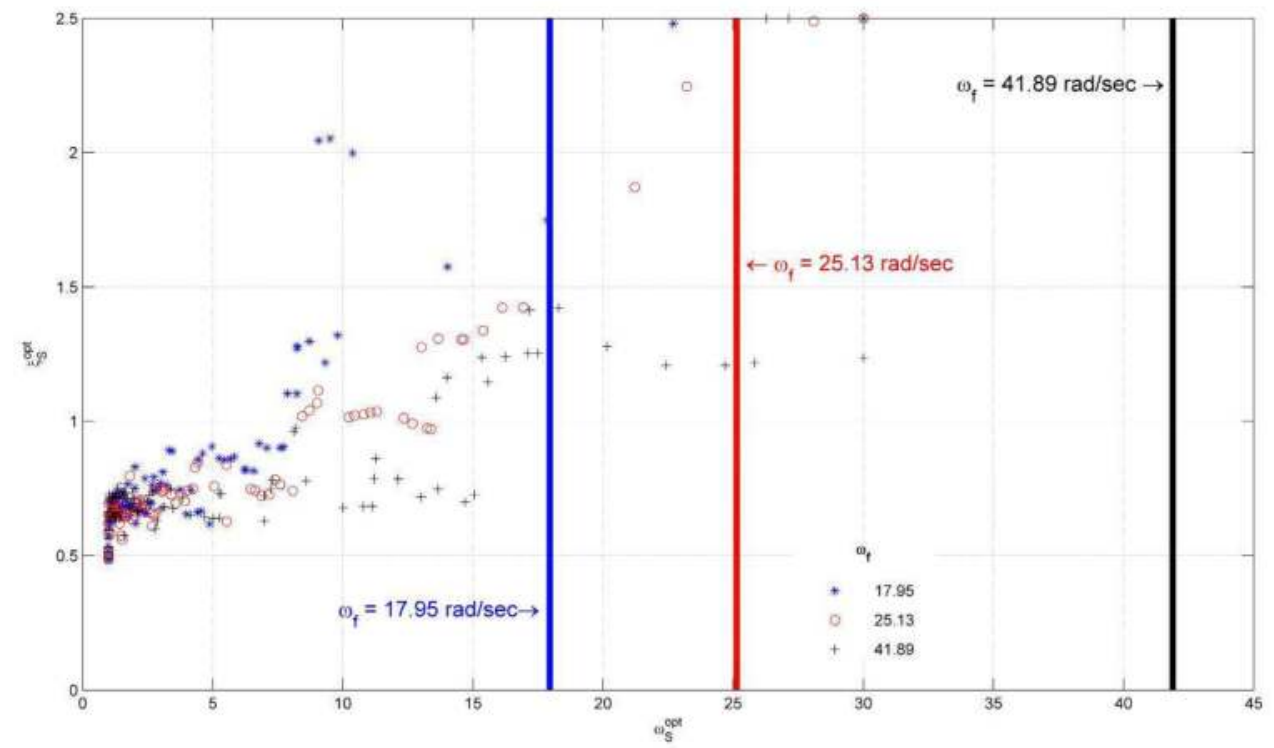

Fig. 10. Space of DV elements of multi-objective problem for different values of filter pulsation. 
Moreover, one can deduce that the variability of both input parameters modify the Pareto set, but the excitation frequency $\omega_{f}$ influences the optimum solution more than $\xi_{f}$. Actually, from figure 9 it is possible to notice that the maximum performance of TMD changes as $\omega_{f}$ varies. Moreover, the initial slopes (for very small admissible displacement) are quite different. In detail, the variation of $O F_{1}$ is greater for higher values of $\omega_{f}$ and tends to decrease as this parameter grows up. Also the optimization strategy in terms of optimum design variables changes (fig. 10). On the left portion of $D V$ space only little variations of optimum $D V$ take place, whereas they correspond to the points located at the bottom on the right of Pareto front in figure 9. These values correspond to the asymptotic value of $O F_{2}$, where the minimum is attained for each displacement. So that, they tend to be located in a small region of the $D V$ space, quite closer to this unconditional optimum solution point.

\section{Conclusions}

In the present study a multi-objective optimization design criterion for linear viscous elastic vibration control devices has been proposed. More in detail, the problem of an isolator device for the vibration control of a single rigid mass have been analysed.

The analysis has been carried out by adopting a stochastic approach, by assuming that the excitations acting on the base of the protected systems are stationary stochastic coloured processes.

In the multi-objective optimization problems two antithetic objectives are considered: the maximization of control strategy performance, expressed in stochastic terms by means of the reduction of transmitted acceleration in the protected systems, and the limitation in stochastic terms of the displacement of the vibrations control device. The design variables are the mechanical characteristics - frequency and damping ratio- of the device.

In order to perform the stochastic multi-objective optimization, the non dominated sorting genetic algorithm in its second version (NSGA-II) has been adopted, which supplies the Pareto set and the corresponding optimum design variables for different system and input configurations.

The sensitivity analysis carried out has showed that the optimum solution (i.e. the maximization of control strategy, expressed in terms of reduction of the response of the main system, and the limitation of the device displacement) is reached, in the two analysed problems, by adopting different strategies, in function of input and system characterization. These strategies act by varying the optimum frequency and damping ratio of the device differently, in function of the allowable performance.

The novelty of the proposed method is in using a multi-dimensional criterion for the design. Nowadays, this is a very important issue in modern Technical Codes [20], in which several performance requirements, which often can conflict each others, are fixed. In these situations, the designer must select the design variables which make available all objectives and the use of a multi-dimension criterion is very useful in this context.

The validation of the proposed method is demonstrated by developing two applications, in which several parameters involved have been changed. Therefore, results attained by the proposed method can be utilised in order to support the designers in the definition of possible structural solutions in vibration control strategy by using linear viscous-elastic devices. 


\section{References}

[1] Lutes L. D., Sarkani S., “Random vibrations”, Butterworth-Heinemann, Oxford (UK), 2001.

[2] Nigam, N.C., "Structural Optimization in Random Vibration Environment", AIAA, pp.551-553, 1972.

[3] Constantinou M. C., Tadjbakhsh I.G., "Optimum design of a first story damping system", Computer and Structures, Vol.17, pp. 305- 310, 1983.

[4] Takewaki I., "An approach to stiffness-damping simultaneous optimization", Computer Methods in Applied Mechanics and Engineering, Vol. 189(2), pp. 641$650,2000$.

[5] Park K. S., Koh H. M., Hahm D., “Integrated optimum design of viscoelastically damped structural systems", Engineering Structures, Vol.26, pp. 581-591, 2004.

[6] Rundinger F., "Optimum vibration absorber with nonlinear viscous power law damping and white noise excitation", ASCE, Journl of Engineering Mechanics, Vol. 132 (1), pp. 46-53, 2006.

[7] Hoang N., Warnitchai P., "Design of multiple tuned mass dampers by using a numerical optimizer", Earthquake Engineering and Structural Dynamic, Vol. 34(2), pp. 125-144, 2005.

[8] Papadrakakis M., Lagaros N. D., Plevris V., "Multiobjective Optimization of skeletal structures under static and seismic loading conditions, Engineering Optimization, Vol. 34, pp. 645-669, 2002.

[9] Tajimi H., "A statistical method of determining the maximum response of a building during earthquake", Proceedings of $2^{\text {nd }}$ World Conf. on Earthquake Engineering, Tokyo, Japan, 1960.

[10] Lin C.C., Wang J.F., Ueng J.M., "Vibration Control identification of seismically excited m.d.o.f structure-PTMD systems", Journal of Sound and Vibration, Vol.240(1), pp. 87-115, 2001.

[11] Crandal S. H., Mark W. D., "Random vibration in mechanical systems", Accademic Press. NY and London, 1963.

[12] C. A. Coello Coello, "Handling Preferences in Evolutionary Multiobjective Optimization: A Survey", IEEE Neural Networks Council (ed.), Proceedings of the 2000 Congress on Evolutionary Computation (CEC 2000) Vol. 1, IEEE Service Center, Piscataway, New Jersey, pp. 30 -37, 2000.

[13] G. C. Luh, C. H. Chuen, "Multi-Objective optimal design of truss structure with immune algorithm", Computers and Structures, Vol. 82, pp. 829-844, 2004.

[14] C. M. Fonseca, P. J. Fleming, "Genetic Algorithms for Multi-Objective Optimization: Formualtion, Discussion and Generalization", Genetic Algorithms: Proceedings of the $5^{\text {th }}$ International Conference (S. Forrest, ed.) San Mateo, CA: Morgan Kaufmann, 1993.

[15] N. Srinivas, K. Deb, "Multi-objective Optimization Using Nondominated Sorting in Genetic Algorithms", Journal of Evolutionary Computation, Vol. 2 (39), pp. 221248, 1994.

[16] K. Deb, S. Agrawal, A. Pratap, T. Meyarivan, “A Fast Elitism Multi-Objective Genetic Algorithm: NSGA-II", Proceedings of Parallel Problem Solving from Nature, Sprinter pp. 849-858, 2000. 
[17] M. M. Raghuwanshi, O. G. Kakde, "Survey on multiobjective evolutionary and real coded genetic algorithms", Proceedings of the $8^{\text {th }}$ Asia Pacific Symposium on Intelligent and Evolutionary Systems, pp. 150-161, 2004.

[18] T. Blickle, L. Thiele, "A Mathematical Analysis of Tournament Selection", in L. Eschelman, ed., Genetic Algorithms: Proceedings of the $6^{\text {th }}$ International Conference (ICGA95), Morgan Kaufmann, San Francisco, CA, 1995.

[19] K. Deb, R. B. Agrawal, "Simulated binary crossover for continuous search space", Complex System, Vol. 9, pp.115-148, 1995.

[20] SEAOC, "Vision 2000: Performance-Based Seismic Engineering of Buildings, Structural Engineers Association of California, Sacramento, California, 1995. 


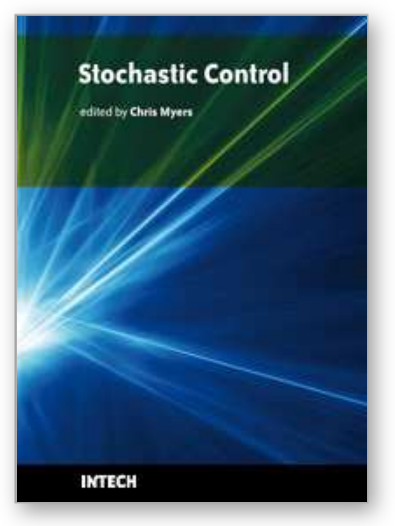

\author{
Stochastic Control \\ Edited by Chris Myers
}

ISBN 978-953-307-121-3

Hard cover, 650 pages

Publisher Sciyo

Published online 17, August, 2010

Published in print edition August, 2010

Uncertainty presents significant challenges in the reasoning about and controlling of complex dynamical systems. To address this challenge, numerous researchers are developing improved methods for stochastic analysis. This book presents a diverse collection of some of the latest research in this important area. In particular, this book gives an overview of some of the theoretical methods and tools for stochastic analysis, and it presents the applications of these methods to problems in systems theory, science, and economics.

\title{
How to reference
}

In order to correctly reference this scholarly work, feel free to copy and paste the following:

Sara Sgobba and Giuseppe Carlo Marano (2010). Optimal Design Criteria for Isolation Devices in Vibration Control, Stochastic Control, Chris Myers (Ed.), ISBN: 978-953-307-121-3, InTech, Available from: http://www.intechopen.com/books/stochastic-control/optimal-design-criteria-for-isolation-devices-in-vibrationcontrol

\section{INTECH}

open science | open minds

\section{InTech Europe}

University Campus STeP Ri

Slavka Krautzeka 83/A

51000 Rijeka, Croatia

Phone: +385 (51) 770447

Fax: +385 (51) 686166

www.intechopen.com

\section{InTech China}

Unit 405, Office Block, Hotel Equatorial Shanghai

No.65, Yan An Road (West), Shanghai, 200040, China 中国上海市延安西路65号上海国际贵都大饭店办公楼 405 单元

Phone: +86-21-62489820

Fax: $+86-21-62489821$ 
(C) 2010 The Author(s). Licensee IntechOpen. This chapter is distributed under the terms of the Creative Commons Attribution-NonCommercialShareAlike-3.0 License, which permits use, distribution and reproduction for non-commercial purposes, provided the original is properly cited and derivative works building on this content are distributed under the same license. 